\title{
MODELING CUSTOMER-EMPLOYEE INSTANT RAPPORT IN THE FIRST SERVICE ENCOUNTER
}

\author{
Jiun-Sheng Chris Lin, National Taiwan University, Taiwan \\ Cheng-Yu Lin, National Taiwan University, Taiwan \\ Wei-Lin Wang, McGill University, Canada
}

\begin{abstract}
A service employee's interaction with a customer in the initial encounter is critically important in creating the customer's impression of the firm and thereby his/her desire to have future interactions with the firm, yet existing marketing literature lacks an understanding of the role instant rapport plays in service delivery. This study attempts to fill the research gap, proposing and testing a model that explores the mechanisms of customer-employee instant rapport development and the resulting service outcomes.

Two hundred and fifty-seven employee-customer pairs, from the apparel retailing industry, participated in this research. Results show that employee affective delivery and connecting behaviors have positive influence on customer positive emotion and instant rapport, which in turns affects customer satisfaction, word of mouth and actual purchase. Results also show that employee's authenticity strengthens the positive relationship between employee's affective delivery and customer positive emotion as well as the positive relationship between employee's affective delivery and customer-employee instant rapport. Implications, limitations and future research directions are then discussed.
\end{abstract}

References available upon request 